\title{
Nomograms Predict Overall Survival and Cancer-Specific Survival in Patients with Fibrosarcoma: A SEER-Based Study
}

\author{
Guang-Heng Xiang $\mathbb{D},{ }^{1,2}$ Juan-Juan Zhu, ${ }^{3}$ Chen-Rong Ke, ${ }^{1}$ Yi-Min Weng, \\ Ming-Qiao Fang, ${ }^{1,2}$ Si-Pin Zhu, ${ }^{1}$ Yu-An Li, ${ }^{1}$ Jian Xiao $\mathbb{D}^{1,2}$ and Lei Xu ${ }^{4}{ }^{4}$ \\ ${ }^{1}$ Department of Orthopaedic, The Second Affiliated Hospital and Yuying Children's Hospital of Wenzhou Medical University, \\ Wenzhou, Zhejiang 325035, China \\ ${ }^{2}$ School of Pharmaceutical Sciences, Wenzhou Medical University, Wenzhou, Zhejiang 325035, China \\ ${ }^{3}$ Department of Geriatrics and Neurology, \\ The Second Affiliated Hospital and Yuying Children's Hospital of Wenzhou Medical University, Wenzhou, \\ Zhejiang 325035, China \\ ${ }^{4}$ Department of Pediatric Surgery, \\ The Second Affiliated Hospital and Yuying Children's Hospital of Wenzhou Medical University, Wenzhou, \\ Zhejiang 325035, China
}

Correspondence should be addressed to Jian Xiao; wenzhouykdx@163.com and Lei Xu; wzykdxwym@163.com

Received 7 June 2020; Revised 25 August 2020; Accepted 12 September 2020; Published 26 September 2020

Academic Editor: Pierfrancesco Franco

Copyright (c) 2020 Guang-Heng Xiang et al. This is an open access article distributed under the Creative Commons Attribution License, which permits unrestricted use, distribution, and reproduction in any medium, provided the original work is properly cited.

\begin{abstract}
Purpose. Due to the rarity, it is difficult to predict the survival of patients with fibrosarcoma. This study aimed to apply a nomogram to predict survival outcomes in patients with fibrosarcoma. Methods. A total of 2235 patients with diagnoses of fibrosarcoma were registered in the Surveillance, Epidemiology, and End Results database, of whom 663 patients were eventually enrolled. Univariate and multivariate Cox analyses were used to identify independent prognostic factors. Nomograms were constructed to predict 3-year and 5-year overall survival and cancer-specific survival of patients with fibrosarcoma. Results. In univariate and multivariate analyses of OS, age, sex, race, tumor stage, pathologic grade, use of surgery, and tumor size were identified as independent prognostic factors. Age, sex, tumor stage, pathologic grade, use of surgery, and tumor size were significantly associated with CSS. These characteristics were further included to establish the nomogram for predicting 3-year and 5 -year OS and CSS. For the internal validation of the nomogram predictions of OS and CSS, the C-indices were 0.784 and 0.801. Conclusion. We developed the nomograms that estimated 3-year and 5-year OS and CSS. These nomograms not only have good discrimination performance and calibration but also provide patients with better clinical benefits.
\end{abstract}

\section{Introduction}

Fibrosarcoma (FS) refers to a specific entity, which is by definition a diagnosis of exclusion, on the basis of the WHO classification of tumors of soft tissue and bone [1]. Fibrosarcoma belongs to the sarcoma cancer group and is a rare and highly malignant tumor of mesenchymal origin. Fibrosarcoma can occur in any anatomical location. It does not directly produce bone or cartilage but forms a primary or secondary bone tumor [2]. Central fibrosarcoma is caused by fibrous tissue in the medullary canal, but periosteal fibrosarcoma results from periosteal connective tissue [3]. Fibrosarcoma includes infant (congenital) fibrosarcoma and adult fibrosarcoma, and adult fibrosarcoma is defined as "malignant neoplasm composed of fibroblasts with variable collagen production and, in classical cases, a "herringbone" architecture" [1]. The prognosis of adult fibrosarcoma is much worse than infant fibroids. In the past period, the incidence of FS has dropped dramatically, and recent data indicate that it accounts for only $3.6 \%$ of sarcomas [4]. Surgery is the standard treatment of patients with localized 


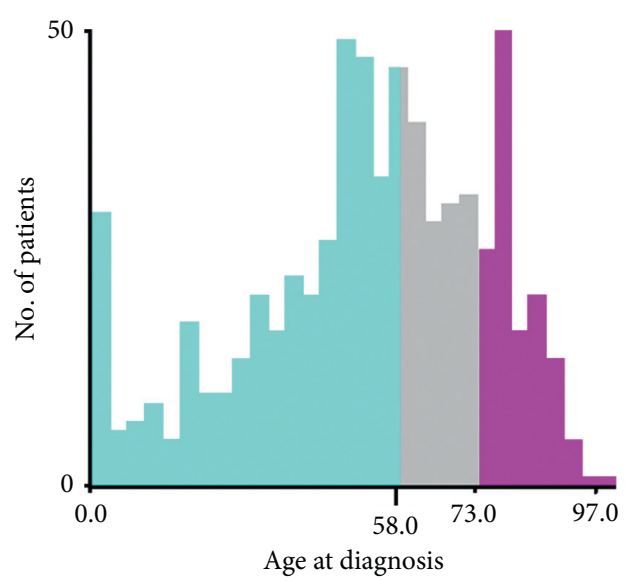

(a)

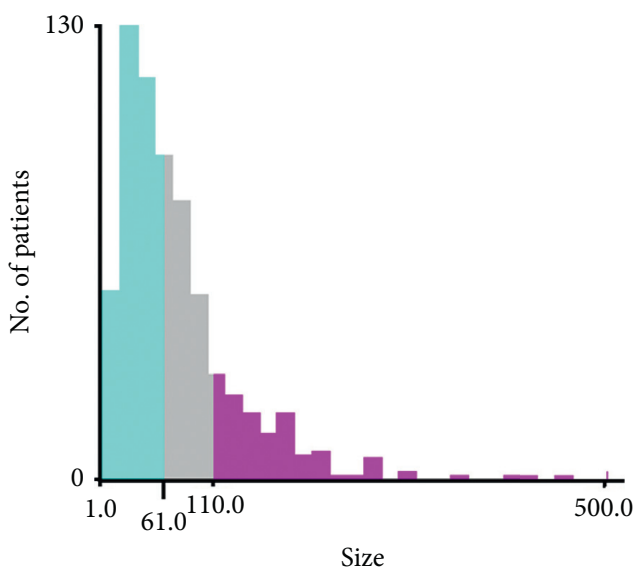

(c)

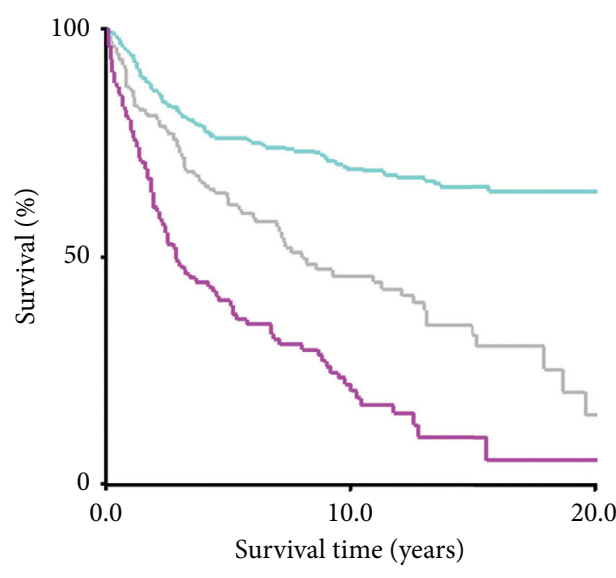

(b)

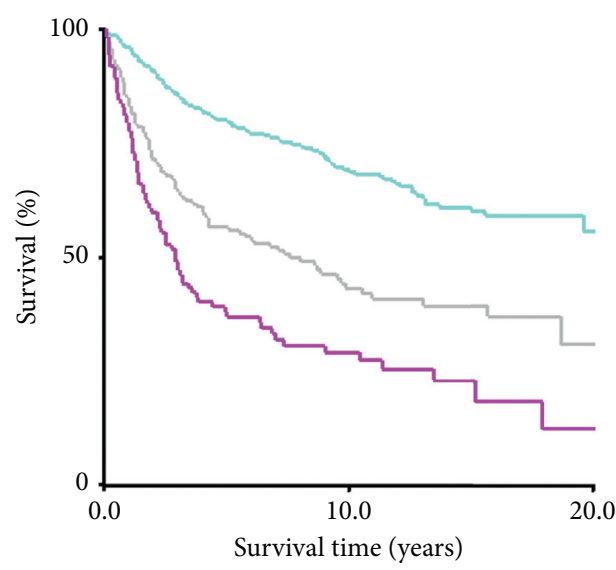

(d)

Figure 1: Identification of optimal cuto values of age of diagnosis and tumor size via $X$-tile analysis. Optimal cuto values of age were identified as 58 and 73 years based on overall survival (a). Optimal cuto values of tumor size were identified as $61 \mathrm{~mm}$ and $110 \mathrm{~mm}$ based on overall survival (c). Histogram and Kaplan-Meier analysis were developed based on these cuto values (b) and (d).

FS. In addition, adjuvant chemotherapy, radiotherapy, and/ or hyperthermia can be discussed in some cases [5].

As with other sarcomas, the overall prognosis of fibrosarcoma depends on tumor size, tumor nature, tumor grade, and the presence or absence of distant metastases $[1,6-9]$. The prognostic factors associated with survival are numerous and complex, and it is meaningful to establish a model to accurately predict the survival outcome of FS. Prognostic nomograms have proven to be an effective method for accurately predicting survival outcomes in cancer patients such as lung cancer, rectal cancer, and gastric cancer patients [10-12]. However, to the best of our knowledge, there is no report on the use of nomograms to predict FS survival outcomes. Therefore, the goal of our study is to identify independent factors that influence the overall survival (OS) and cancer-specific survival (CSS) of patients with FS and then establish a nomogram to accurately predict the incidence of OS and CSS at 3-year and 5 -year.

\section{Materials and Methods}

2.1. Patients Source and Selection. The Surveillance, Epidemiology, and End Results (SEER) registry program provides information on cancer statistics since 1973. This database covering approximately $28 \%$ of the population in 18 different regions of the United States is supported by the Surveillance Research Program in National Cancer Institute Division of Cancer Control and Population Sciences. The SEER $*$ Stat software Version 8.3.6 (https://seer.cancer. gov/seerstat/, NCI, Bethesda, USA) provides a convenient, intuitive mechanism for the analysis of SEER and other cancer-related databases.

The inclusion criteria for this study were as follows: (a) patients were diagnosed as $8811 / 3,8812 / 3,8813 / 3$, and $8814 /$ 3 in ICD-O-3 (International Classification of Disease for Oncology, 3rd Edition); (b) histological examination confirmed as FS; (c) completed the follow-up period; and (d) complete survival and death date. Exclusion criteria in this 
TABLE 1: Patient cohort characteristics.

\begin{tabular}{lc}
\hline Variables & Value, $n(\%)$ \\
\hline Age, years & \\
$<58$ & $370(55.8 \%)$ \\
$58-73$ & $158(23.8 \%)$ \\
$>73$ & $135(20.4 \%)$ \\
\hline Sex & \\
Male & $332(50.1 \%)$ \\
Female & $331(49.9 \%)$ \\
\hline Race & \\
White & $522(78.7 \%)$ \\
Black & $92(13.9 \%)$ \\
Others & $49(7.4 \%)$ \\
\hline Surgery & \\
Yes & $620(93.5 \%)$ \\
No & $43(6.5 \%)$ \\
\hline Marital status & \\
Married & $345(52.0 \%)$ \\
Unmarried & $318(48.0 \%)$ \\
\hline Stage & \\
Localized & $413(62.3 \%)$ \\
Regional & $188(28.4 \%)$ \\
Distant & $62(9.3 \%)$ \\
\hline Grade & \\
I & $156(23.5 \%)$ \\
II & $222(33.5 \%)$ \\
III & $132(19.9 \%)$ \\
IV & $153(23.1 \%)$ \\
\hline Tumor size (mm) & $354(53.4 \%)$ \\
$<61$ & $184(27.7 \%)$ \\
$61-110$ & $125(18.9 \%)$ \\
$>110$ &
\end{tabular}

study were as follows: (a) only autopsy or death certificate; (b) the data of tumor size, pathologic grade, or stage missing; and (c) the data of surgery treatment missing.

2.2. Patients Variables. The information about clinicopathological features, including age at diagnosis, year of diagnosis, race, sex, histology, pathologic grade, disease stage, tumor size, use of surgery, marital status, vital status, and months of survival, were recorded in our study. The race was divided into white, black, and others. Marital status was categorized as married and unmarried. The pathological grade was divided into four categories according to the "ICD-O-3 grade": grades I, II, III, and IV. According to the American Joint Committee on Cancer (AJCC) staging system, the FS tumor stage was divided into local, regional, and distant (Supplementary Figure S1).

2.3. Statistical Analysis. SPSS 23.0 (IBM Corp.) was used to assess the patient variables collected from the SEER databases. The two main endpoints of this study were overall survival and cancer-specific survival. Overall survival was defined as the period from diagnosis to death from any disease cause. Cancerspecific survival was defined as the period from diagnosis to death from FS. The cuto value of age at diagnosis and tumor size was calculated by $X$-tile software (Yale University, New Haven, Connecticut, USA), according to OS (Figure 1). We constructed
TABLE 2: Univariate and multivariate analyses of variables associated with overall survival.

\begin{tabular}{|c|c|c|c|}
\hline \multirow{2}{*}{ Variables } & \multirow{2}{*}{$\begin{array}{c}\text { Univariate } \\
\text { analysis } \\
P \text { value }\end{array}$} & \multicolumn{2}{|c|}{ Multivariate analysis } \\
\hline & & HR $(95 \% \mathrm{CI})$ & $P$ value \\
\hline \multicolumn{4}{|l|}{ Age, years } \\
\hline$<58$ & \multirow{3}{*}{$<0.001$} & Reference & \\
\hline $58-73$ & & $2.200(1.622-2.986)$ & $<0.001$ \\
\hline$>73$ & & $3.902(2.911-5.230)$ & $<0.001$ \\
\hline \multicolumn{4}{|l|}{ Sex } \\
\hline Male & \multirow[t]{2}{*}{0.039} & Reference & \\
\hline Female & & $0.756(0.593-0.963)$ & 0.024 \\
\hline \multicolumn{4}{|l|}{ Race } \\
\hline White & \multirow{3}{*}{0.003} & Reference & \\
\hline Black & & $0.814(0.547-1.211)$ & 0.310 \\
\hline Others & & $0.636(0.361-1.121)$ & 0.118 \\
\hline \multicolumn{4}{|l|}{ Surgery } \\
\hline Yes & \multirow{2}{*}{$<0.001$} & Reference & \\
\hline No & & $3.073(1.993-4.738)$ & $<0.001$ \\
\hline \multicolumn{4}{|l|}{ Marital status } \\
\hline Married & \multirow[t]{2}{*}{0.0726} & Reference & \\
\hline Unmarried & & $0.836(0.651-1.075)$ & 0.163 \\
\hline \multicolumn{4}{|l|}{ Stage } \\
\hline Localized & \multirow{3}{*}{$<0.001$} & Reference & \\
\hline Regional & & $1.244(0.947-1.635)$ & 0.117 \\
\hline Distant & & $2.660(1.859-3.807)$ & $<0.001$ \\
\hline \multicolumn{4}{|l|}{ Grade } \\
\hline I & \multirow{4}{*}{$<0.001$} & Reference & \\
\hline II & & $1.655(1.116-2.455)$ & 0.012 \\
\hline III & & $1.907(1.261-2.885)$ & 0.002 \\
\hline IV & & $3.000(2.023-4.450)$ & $<0.001$ \\
\hline \multicolumn{4}{|l|}{$\begin{array}{l}\text { Tumor size } \\
(\mathrm{mm})\end{array}$} \\
\hline$<61$ & \multirow[t]{3}{*}{$<0.001$} & Reference & \\
\hline $61-110$ & & $1.931(1.446-2.578)$ & $<0.001$ \\
\hline$>110$ & & $2.784(2.057-3.766)$ & $<0.001$ \\
\hline
\end{tabular}

the cumulative survival curves using the Kaplan-Meier method and compared the variables by a log-rank test. Cox proportional hazard regression analyses were used to determine independent prognostic factors, and the results are presented as hazard ratios and corresponding 95\% confidence intervals (95\% CIs). According to the results obtained by multivariate Cox proportional hazards regression analyses, nomograms for 3-year and 5-year OS and 3-year and 5-year CSS were constructed by applying the rms package in $R$ software, version 3.6.1 (http:// www.r-project.org/). Concordance index ( $C$-index) was used to evaluate the reliability of nomogram [13]. The $C$-index ranged from 0.5 (a poor model) to 1.0 (a perfect model), and it more than 0.7 represented a good predictive performance [14]. The calibration curve was used to compare the conformity between the predicted and actual survival. A two-tailed $P<0.05$ was considered statistically significant.

\section{Results}

A total of 2235 patients diagnoses as FS were enrolled in the SEER database from 1975 to 2016, among them 663 patients eventually participated in our study on the basis of the above 


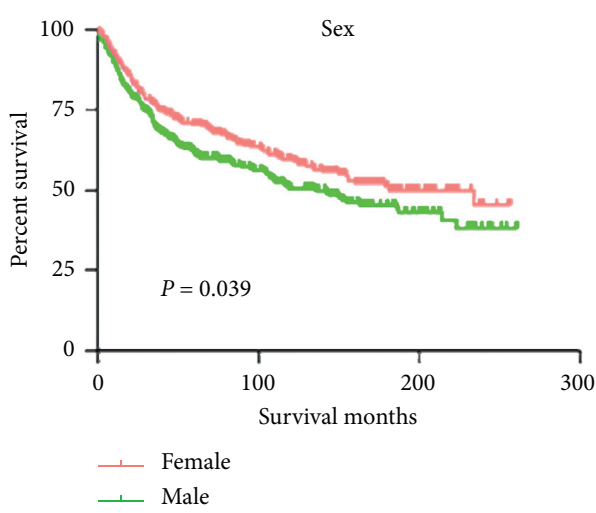

(a)

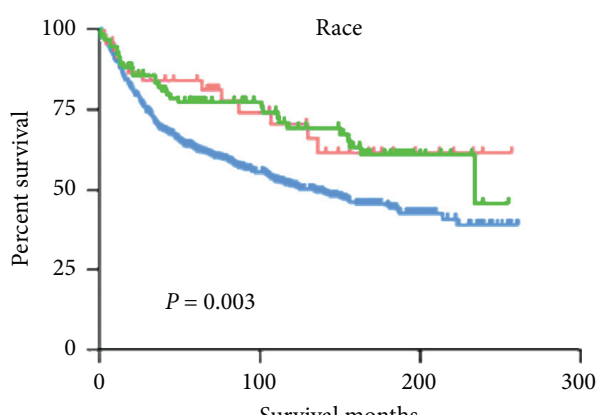

_- Black

- Others

$\rightarrow$ White

(c)

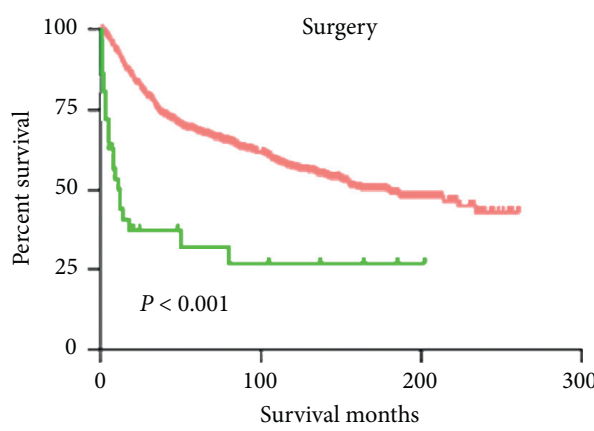

$\longrightarrow$ No

— Yes

(e)

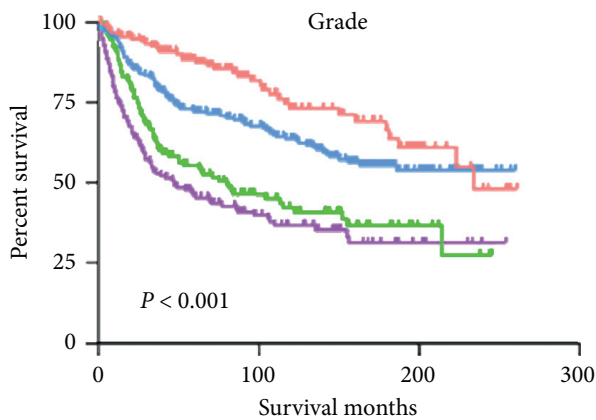

$\begin{array}{ll}-\mathrm{I} & \text { III } \\ \mathrm{II} & \mathrm{II}\end{array}$

(g)

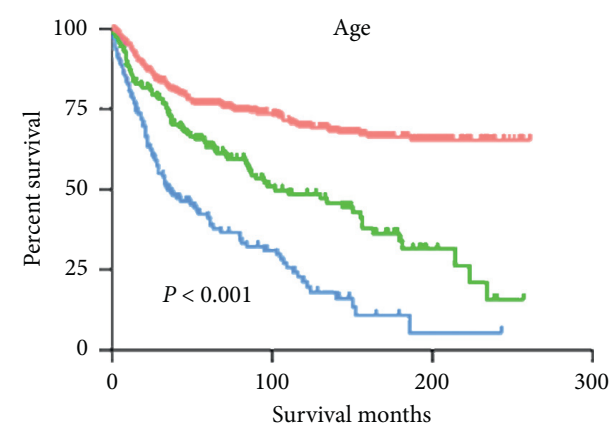

- < 58 years

$+58-73$ years

$+>73$ years

(b)

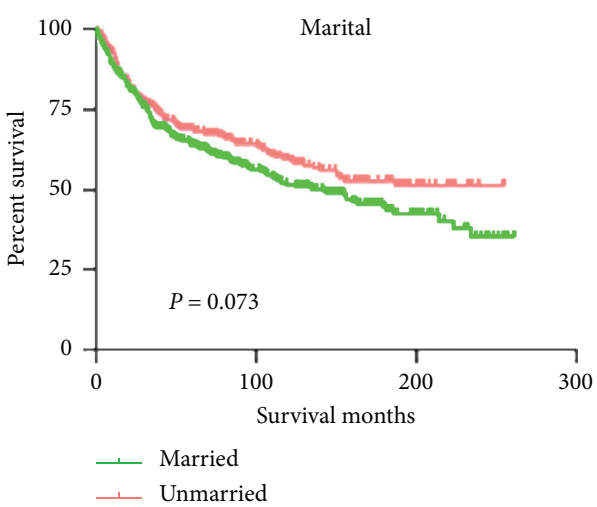

(d)

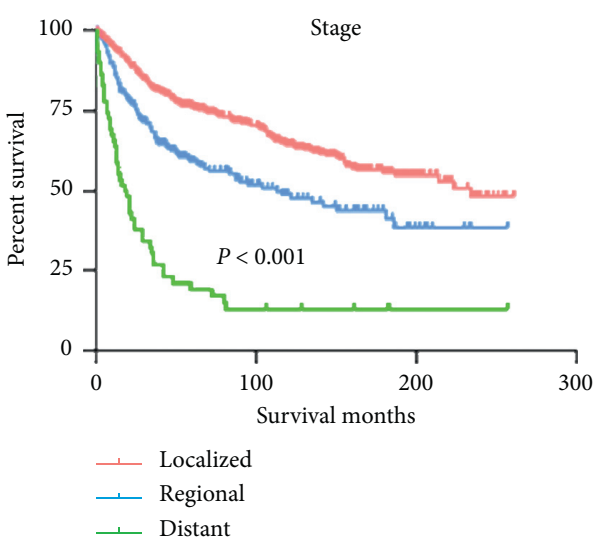

(f)

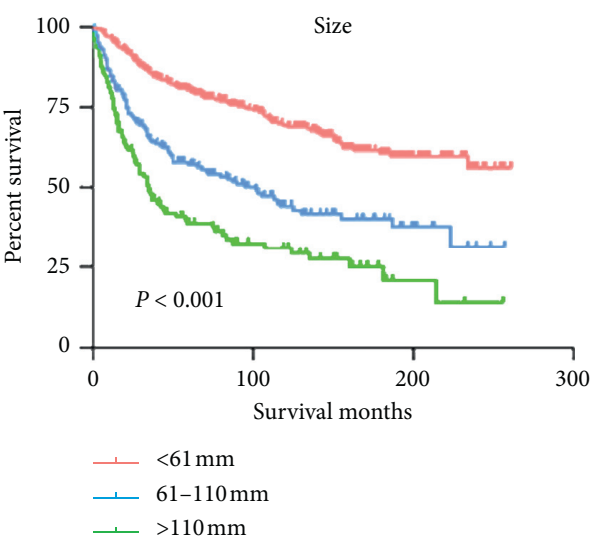

(h)

FIgURe 2: Kaplan-Meier curves of variables were performed for OS in patients with fibrosarcoma. (a) Sex, (b) age, (c) race, (d) marital, (e) use of surgery, (f) tumor stage, (g) tumor grade, and (h) tumor size. 
TABLE 3: Univariate and multivariate analyses of variables associated with cancer-specific death.

\begin{tabular}{|c|c|c|c|}
\hline \multirow{2}{*}{ Variables } & \multirow{2}{*}{$\begin{array}{c}\text { Univariate analysis } \\
P \text { value }\end{array}$} & \multicolumn{2}{|c|}{ Multivariate analysis } \\
\hline & & $\operatorname{HR}(95 \% \mathrm{CI})$ & $P$ value \\
\hline \multicolumn{4}{|l|}{ Age, years } \\
\hline$<58$ & \multirow{3}{*}{0.049} & Reference & \\
\hline $58-73$ & & $1.353(0.905-2.022)$ & 0.140 \\
\hline$>73$ & & $1.382(0.892-2.139)$ & 0.147 \\
\hline \multicolumn{4}{|l|}{ Sex } \\
\hline Male & \multirow{2}{*}{0.011} & Reference & \\
\hline Female & & $0.697(0.494-0.984)$ & 0.040 \\
\hline \multicolumn{4}{|l|}{ Race } \\
\hline White & \multirow{3}{*}{0.070} & Reference & \\
\hline Black & & $0.704(0.411-1.206)$ & 0.201 \\
\hline Others & & $0.466(0.189-1.149)$ & 0.097 \\
\hline \multicolumn{4}{|l|}{ Surgery } \\
\hline Yes & \multirow{2}{*}{$<0.001$} & Reference & \\
\hline No & & $3.470(1.994-6.039)$ & $<0.001$ \\
\hline \multicolumn{4}{|l|}{ Marital status } \\
\hline Married & \multirow[t]{2}{*}{0.492} & & \\
\hline Unmarried & & & \\
\hline \multicolumn{4}{|l|}{ Stage } \\
\hline Localized & \multirow{3}{*}{$<0.001$} & Reference & \\
\hline Regional & & $1.788(1.206-2.650)$ & 0.004 \\
\hline Distant & & $4.269(2.656-6.860)$ & $<0.001$ \\
\hline \multicolumn{4}{|l|}{ Grade } \\
\hline I & \multirow{4}{*}{$<0.001$} & Reference & \\
\hline II & & $1.753(0.956-3.215)$ & 0.070 \\
\hline III & & $1.716(0.921-3.198)$ & 0.089 \\
\hline IV & & $3.321(1.847-5.972)$ & $<0.001$ \\
\hline
\end{tabular}

\begin{tabular}{lccc}
\hline $\begin{array}{l}\text { Tumor size } \\
(\mathrm{mm})\end{array}$ & & \\
$<61$ & $<0.001$ & Reference & \\
$61-110$ & & $2.039(1.301-3.195)$ & 0.002 \\
$>110$ & $4.579(2.980-7.035)$ & $<0.001$ \\
\hline
\end{tabular}

inclusion and exclusion criteria. The clinical basic characteristics of patients are shown in Table 1. Among these 663 cases, 331 patients were female $(49.9 \%)$ and 332 patients were male $(50.1 \%)$. The average age of the whole population was 52.65 years. The majority $(78.7 \%)$ of tumors was found in white patients. With regard to the pathologic grades, grade II was the most common $(n=222(33.5 \%))$, followed by grade I $(n=156(23.5 \%))$, grade IV $(n=153(23.1 \%))$, and grade III $(n=132(19.9 \%))$. About the tumor size of FS, $53.4 \%$ of patients had a tumor size less than $61 \mathrm{~mm}, 27.7 \%$ had a tumor between $61 \mathrm{~mm}$ and $110 \mathrm{~mm}$, and $18.9 \%$ had a tumor greater than $110 \mathrm{~mm}$. Regarding tumor staging, $62.3 \%$ of patients had localized disease, $28.4 \%$ patients had regional disease, and the remaining $9.3 \%$ patients had distant disease. For treatment, $93.5 \%$ underwent surgery.

As shown in Table 2, univariate and multivariate analyses of variables were performed for OS in patients with fibrosarcoma. There were seven variables involving patient age $(P<0.001)$, sex $(P=0.039)$, race $(P=0.003)$, tumor stage $(P<0.001)$, pathologic grade $(P<0.001)$, use of surgery $(P<0.001)$, and tumor size $(P<0.001)$ that were related to OS, and the marital had no significant difference (Figure 2).
Further multivariate analyses of important factors identified by univariate analyses were performed, and the results showed that patient age, sex, surgery, tumor stage, pathologic grade, and tumor size were the independent risk factors.

Univariate and multivariate analyses of variables associated with CSS of fibrosarcoma are shown in Table 3. The results of the analyses showed that patient age $(P=0.049)$, sex $(P=0.011)$, tumor stage $(P<0.001)$, pathologic grade $(P<0.001)$, use of surgery $(P<0.001)$, and tumor size $(P<0.001)$ were significantly associated with CSS, while race $(P=0.070)$ and marital $(P=0.492)$ were not significantly correlated with CSS (Figure 3). For the multivariate analyses results, sex, surgery, tumor stage, pathologic grade, and tumor size were determined as independent prognostic factors for CSS.

Independent risk factors determined by multivariate analyses were used to construct the prognostic nomograms for predicting 3-year and 5-year overall survival and cancerspecific survival of patients with fibrosarcoma (Figure 4). The result of OS prediction by nomogram showed that age was the main factor affecting prognosis, followed by surgery, pathological grade, tumor size, tumor stage, and gender, whereas as for CSS, the result showed that tumor size was the most critical factor affecting prognosis, followed by tumor stage, surgery, pathologic grade, and sex. For the internal validation of the nomograms of OS and CSS, the $C$-indices were 0.784 and 0.801 , separately. The calibration plots showed the excellent consistency between the nomogram prediction and the actual survival (Figure 5).

\section{Discussion}

Multiple prognostic factors can affect the survival outcome of cancer patients, while a single prognostic factor cannot fully predict the individual survival. In the light of the scarcity and differences in fibrosarcoma cases, assessing clinical prognosis outcomes can be very challenging. Relying on the traditional AJCC staging system as before has not been sufficient to accurately guide treatment and assess the prognosis of cancer. Nomogram is a graphical illustration of a statistical model for calculating the cumulative effect of several variables and can be used to predict individual survival outcomes. Nomograms have been established for a variety of cancers and have shown more accurate in predicting prognosis than traditional tools [14-19]. As far as we know, the present study is the first article to develop and validate the prognostic nomograms for both OS and CSS in patients with FS. We developed comprehensive nomograms for 3-year and 5-year overall survival and cancer-specific survival on the basis of 663 cases extracted from the SEER database.

Our results showed that the following independent prognostic factors could influence the survival of patients with FS: age, sex, surgery, tumor stage, pathologic grade, and tumor size. The result of OS prediction by nomogram showed that age was the main factor affecting prognosis. It was widely believed that age was related to the survival outcome of various cancers [20-23]. The correlation 


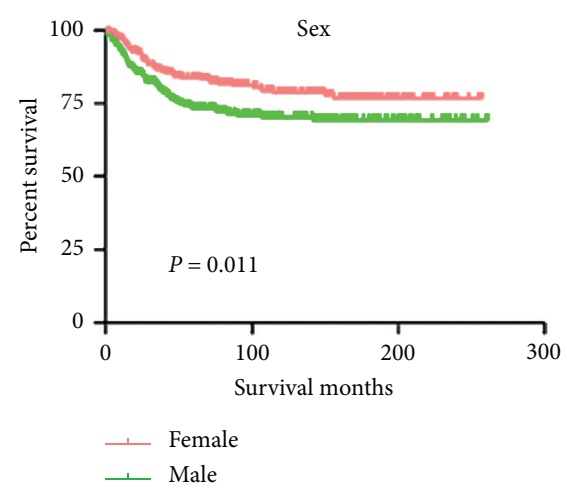

(a)

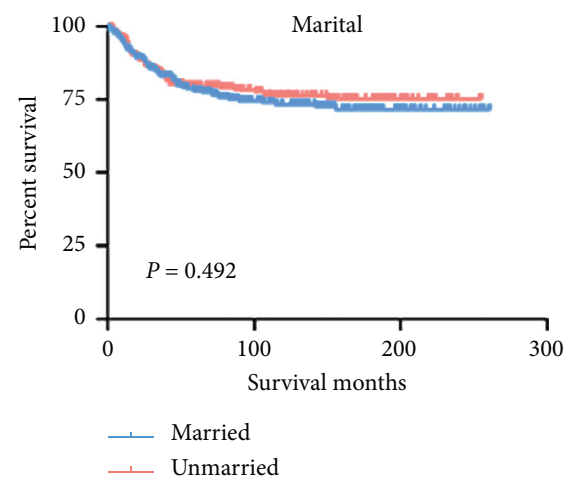

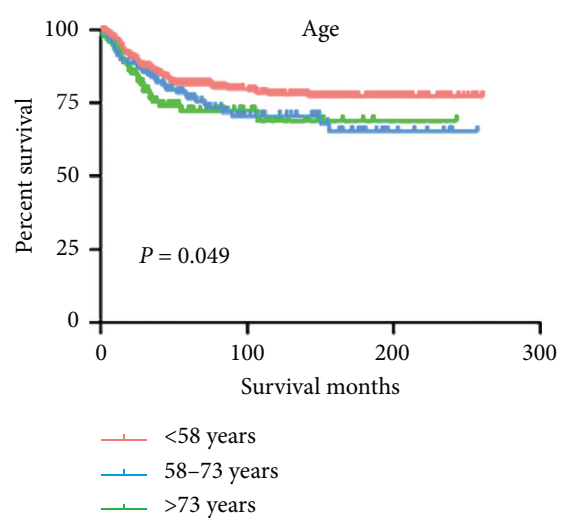

(b)

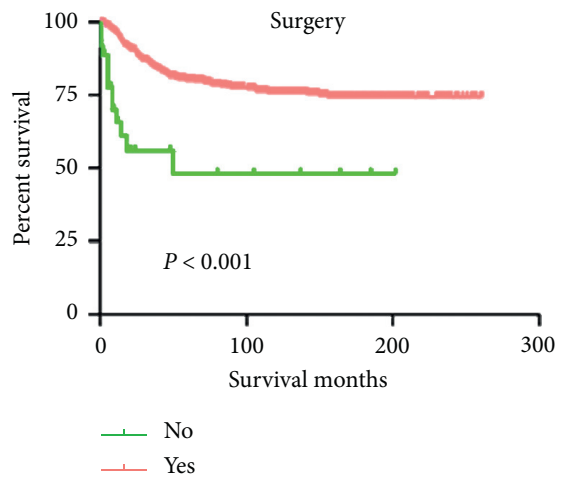

(e)

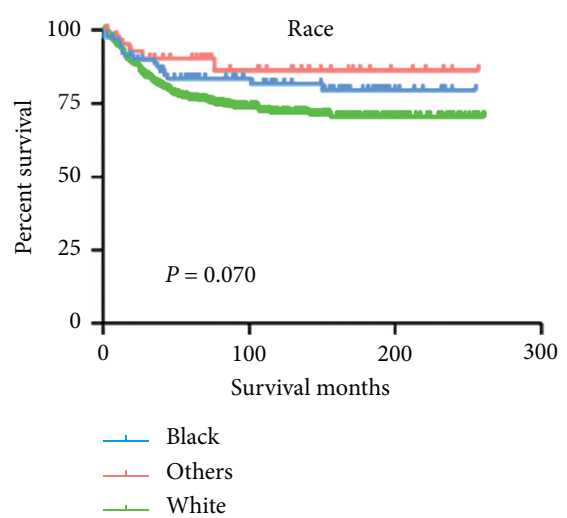

(c)

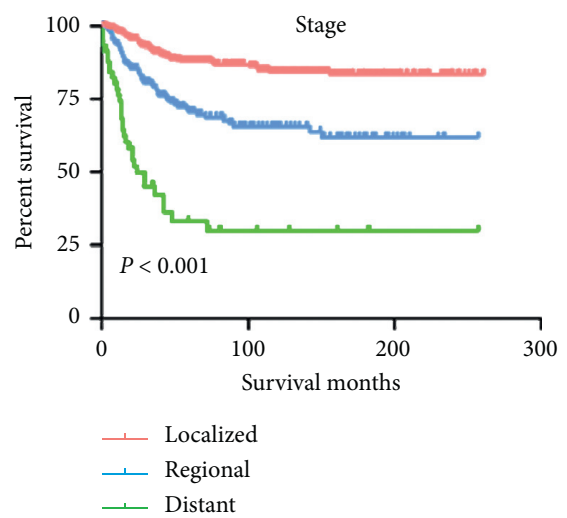

(f)

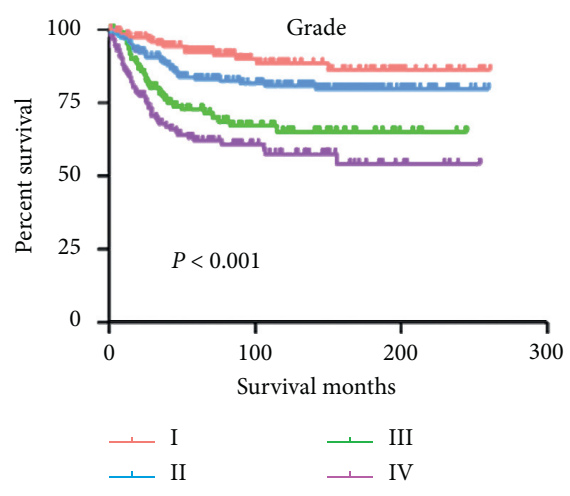

(g)

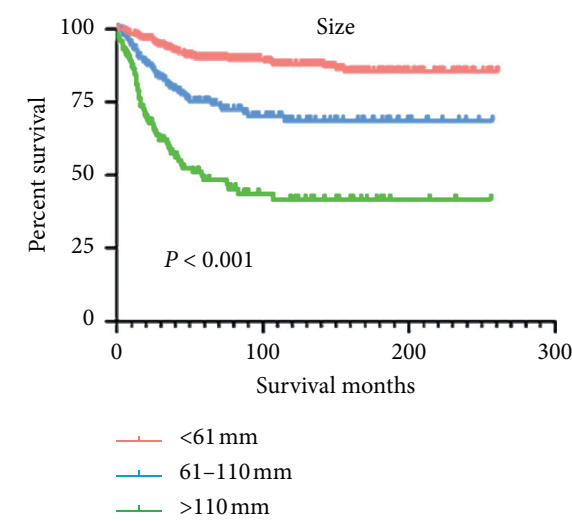

(h)

Figure 3: Kaplan-Meier curves of variables were performed for CSS in patients with fibrosarcoma. (a) Sex, (b) age, (c) race, (d) marital, (e) use of surgery, (f) tumor stage, $(\mathrm{g})$ tumor grade, and $(\mathrm{h})$ tumor size.

between age and OS might be partly due to our use of allcause mortality rather than cancer-specific survival. In other words, older patients usually had chronic diseases or postoperative complications that made them more likely to die.

Although it appears that a larger tumor predicts a poor prognosis, it is necessary to conduct further studies to examine this. In previous studies, the effect of tumor size on survival was inconsistent. Most of these studies believed that larger tumors size was harmful to patient survival [24-27]. In contrast, other studies supported that tumor size had no influence on the survival [28-30]. Regarding our article, the result indicated that tumor size was the most important factor affecting CSS. One possible explanation for these findings was that tumor size during diagnosis was related to the treatment used, which might affect survival.

Multivariate COX regression analysis showed that sex, surgery, tumor stage, and pathologic grade were also independent prognostic indicators for FS patients. Gender was also an important variable related to the prognosis of patients with cancer. In our article, the survival rates of male FS patients were worse than that of female patients. Our study 


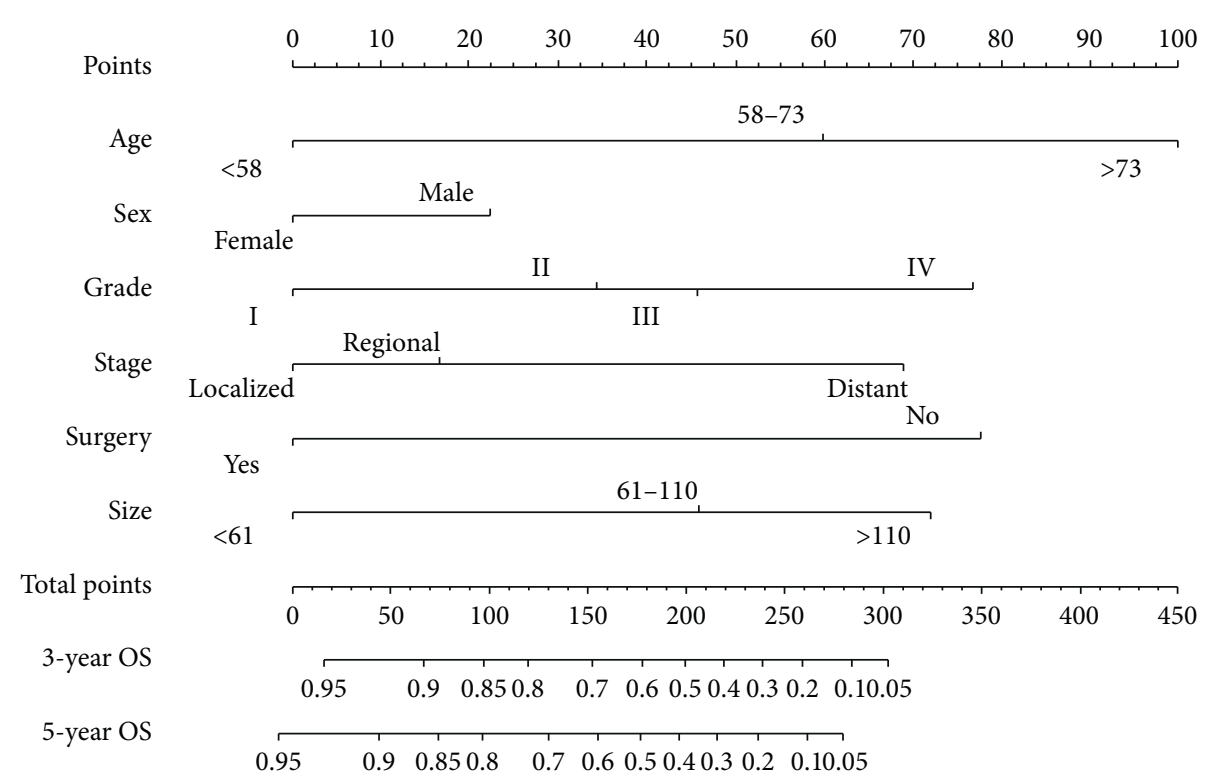

(a)

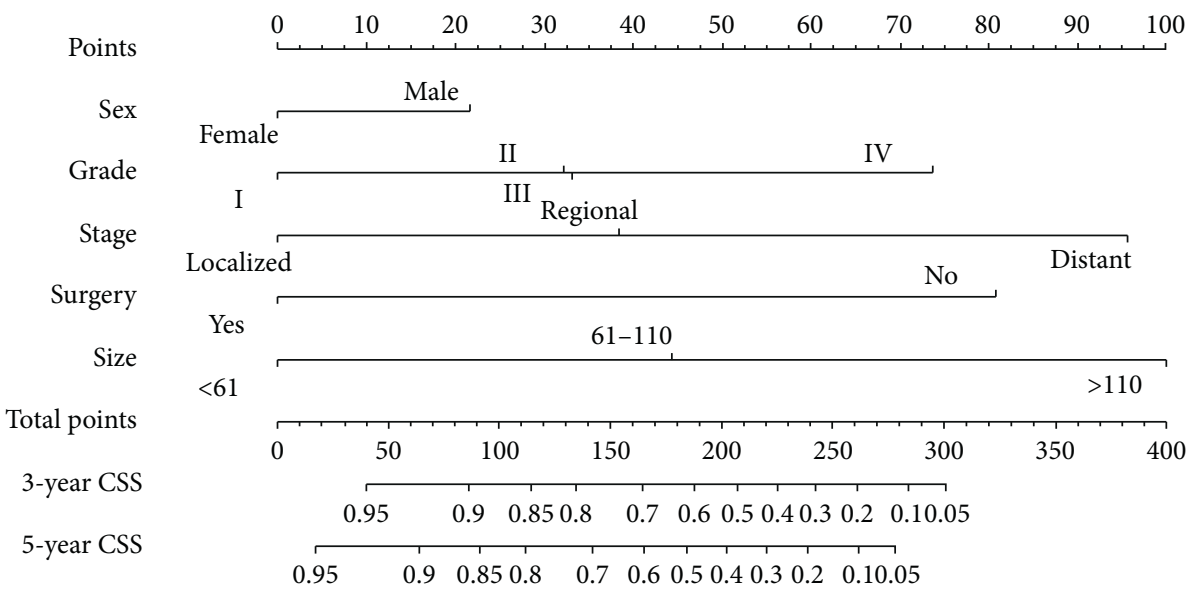

(b)

FIgURE 4: Nomogram to predict overall survival (OS) and cancer-specific survival (CSS) in patients with fibrosarcoma. (a) Predicting 3-year and 5-year OS rates. (b) Predicting 3-year and 5-year CSS rates.

also showed that surgical treatment was related to a better prognosis. Tumor stage was also an independent prognostic factor. The presence of distant stage resulted with a lower survival rate than localized or regional stage. This trend further demonstrated the importance of improving early diagnosis. In addition, pathologic grade could reflect the biological behavior of malignant tumors, which are associated with the occurrence of distant metastasis, and worsened the prognosis of survival outcome.

This study was based on data extracted from the SEER database, which had a large sample size and sufficient cancer data. However, our research had some limitations. As a retrospective study, these findings may be needed to be further validated by randomized controlled trials and prospective studies. Some clinical pathological parameters, such as comorbidities, vascular infiltration, surgical margin status, chemotherapy, or other treatment, were not available in the SEER database, so we did not include these factors in the nomogram [31-34]. Finally, the $C$-index is a good nomogram verification tool, but it is more reliable if you use other independent large-scale datasets for external verification.

\section{Conclusions}

Patient age, sex, use of surgery, tumor stage, pathologic grade, and tumor size were determined as independent prognostic factors of patients with fibrosarcomas. We developed the nomograms that estimated 3-year and 5-year overall survival and cancer-specific survival based on 663 cases extracted from the SEER database. These nomograms not only had good discrimination performance and calibration but also provided patients with better clinical benefits. 


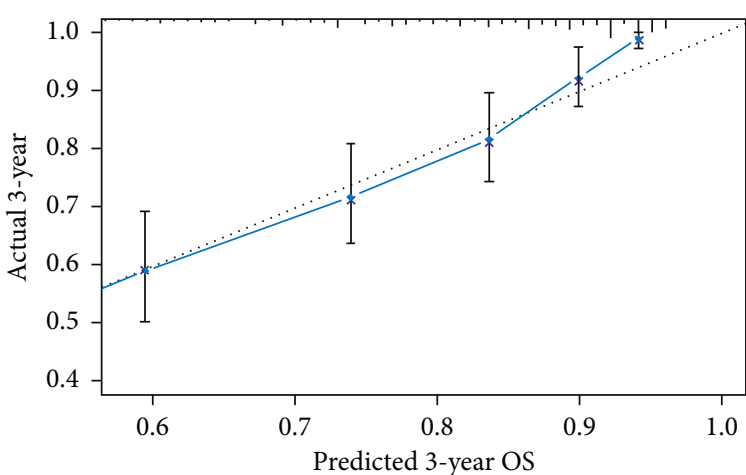

(a)

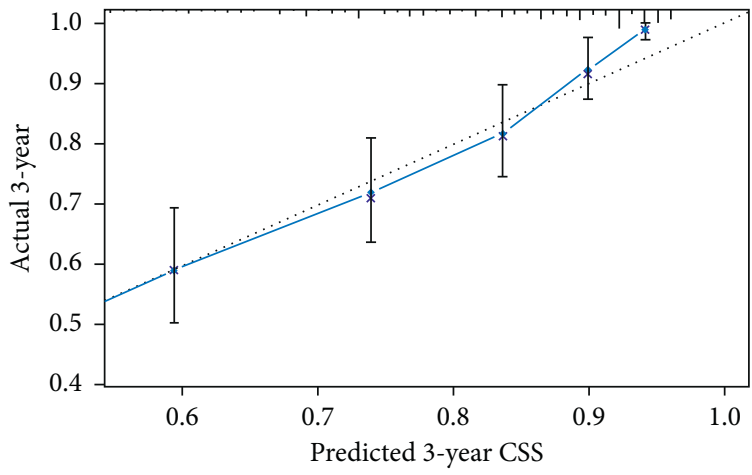

(c)

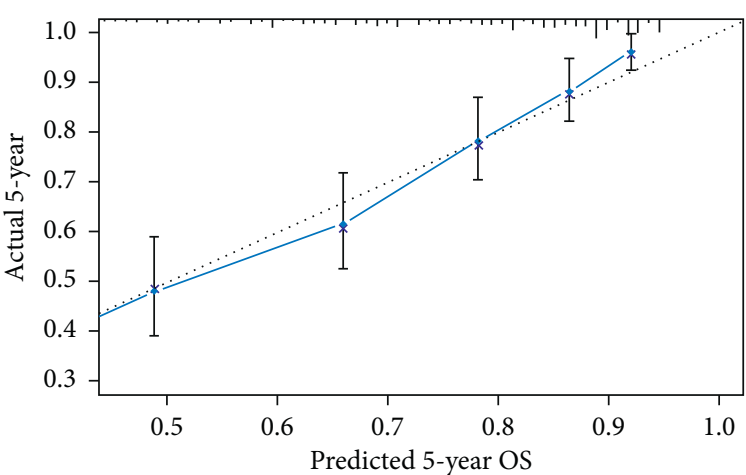

(b)

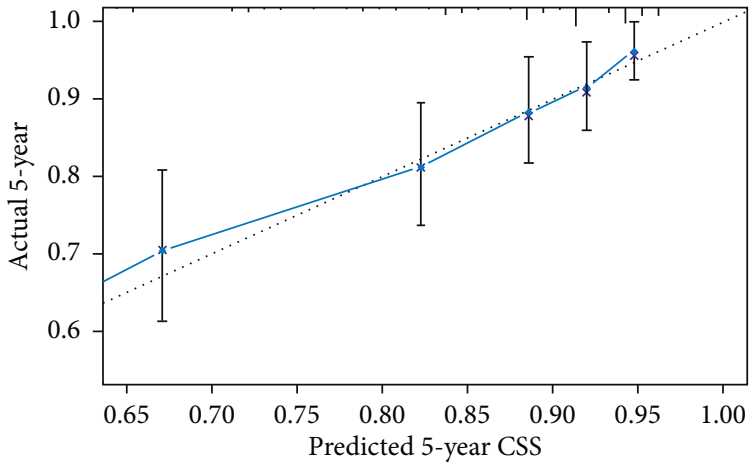

(d)

FIgURE 5: Calibration curves of the nomogram predicting overall survival (OS) and cancer-specific survival (CSS) in patients with fibrosarcoma. (a) 3-year OS rate, (b) 5-year OS rate, (c) 3-year CSS rate, and (d) 5-year CSS rate.

\section{Data Availability}

Publicly available datasets were analyzed in this study. The data used to support this study are available at https://seer. cancer.gov/.

\section{Ethical Approval}

This study was approved by the hospital's ethics committee.

\section{Disclosure}

Statistical analyses were carried out using $R$ version 3.6.1 for Windows ( $R$ Foundation for Statistical Computing, Vienna, Austria), SPSS 23.0 (IBM Corp.), and X-tile software (Yale University, New Haven, Connecticut, USA).

\section{Conflicts of Interest}

The authors declare that they have no conflicts of interest.

\section{Authors' Contributions}

Guang-Heng Xiang, Juan-Juan Zhu, and Si-Pin Zhu contributed to the idea and design. Guang-Heng Xiang, ChenRong Ke, Ming-Qiao Fang, Yu-An Li, and Yi-Min Weng contributed to the data acquisition and analysis. GuangHeng Xiang, Jian Xiao, and Lei $\mathrm{Xu}$ contributed to the manuscript writing and revision. All authors contributed to data acquisition and analysis and to manuscript writing and revision and agreed to all aspects of the work.

\section{Supplementary Materials}

The study flow diagram of the selection process. (Supplementary Materials)

\section{References}

[1] C. D. M. Fletcher, J. A. Bridge, P. C. W. Hogendoorn, and F. Mertens, WHO Classification of Tumours of Soft Tissue and Bone, IARC Press, Lyons, France, 4th edition, 2013.

[2] J. B. Wojcik, A. M. Bellizzi, P. Dal Cin et al., "Primary sclerosing epithelioid fibrosarcoma of bone," The American Journal of Surgical Pathology, vol. 38, no. 11, pp. 1538-1544, 2014.

[3] A. D. Thomson and R. T. Turner-Warwick, "Skeletal sarcomata and giant-cell tumour," The Journal of Bone and Joint Surgery. British volume, vol. 37-B, pp. 266-303, 1955.

[4] J. R. Toro, L. B. Travis, H. J. Wu, K. Zhu, C. D. M. Fletcher, and S. S. Devesa, "Incidence patterns of soft tissue sarcomas, regardless of primary site, in the surveillance, epidemiology and end results program, 1978-2001: an analysis of 26,758 cases," International Journal of Cancer, vol. 119, no. 12, pp. 2922-2930, 2006.

[5] ESMO/European Sarcoma Network Working Group, "Soft tissue and visceral sarcomas: ESMO Clinical Practice Guidelines for diagnosis, treatment and follow-up," Annals of Oncology, vol. 25, pp. iii102-iii112, 2014. 
[6] A. M. Oliveira and A. G. Nascimento, "Grading in soft tissue tumors: principles and problems," Skeletal Radiology, vol. 30, no. 10, pp. 543-559, 2001.

[7] J. N. Cormier and R. E. Pollock, "Soft tissue sarcomas," CA: A Cancer Journal for Clinicians, vol. 54, no. 2, pp. 94-109, 2004.

[8] K. Thway, "Pathology of soft tissue sarcomas," Clinical Oncology, vol. 21, no. 9, pp. 695-705, 2009.

[9] J. M. Coindre, "Grading of soft tissue sarcomas: review and update," Archives of Pathology \& Laboratory Medicine, vol. 130, no. 10, pp. 1448-1453, 2006.

[10] J. Deng, Z. Ren, J. Wen et al., "Construction of a nomogram predicting the overall survival of patients with distantly metastatic non small cell lung cancer," Cancer Management and Research, vol. 10, pp. 6143-6156, 2018.

[11] N. J. Battersby, G. Bouliotis, K. J. Emmertsen et al., "Development and external validation of a nomogram and online tool to predict bowel dysfunction following restorative rectal cancer resection: the POLARS score," Gut, vol. 67, pp. gutjnl-2016, 2018.

[12] S. Y. Kim, M. J. Yoon, Y. I. Park, M. J. Kim, B.-H. Nam, and S. R. Park, "Nomograms predicting survival of patients with unresectable or metastatic gastric cancer who receive combination cytotoxic chemotherapy as first-line treatment," Gastric Cancer, vol. 21, no. 3, pp. 453-463, 2018.

[13] F. E. Harrell Jr., K. L. Lee, and D. B. Mark, "Multivariable prognostic models: issues in developing models, evaluating assumptions and adequacy, and measuring and reducing errors," Statistics in Medicine, vol. 15, no. 4, pp. 361-387, 1996.

[14] W. Liang, L. Zhang, G. Jiang et al., "Development and validation of a nomogram for predicting survival in patients with resected nonsmall cell lung cancer," Journal of Clinical Oncology, vol. 33, no. 8, pp. 861-869, 2015.

[15] Y. Wang, J. Li, Y. Xia et al., "Prognostic nomogram for intrahepatic cholangiocarcinoma after partial hepatectomy," Journal of Clinical Oncology, vol. 31, no. 9, pp. 1188-1195, 2013.

[16] S. Wu, J.-N. Chen, Q.-W. Zhang et al., "A new metastatic lymph node classification-based survival predicting model in patients with small bowel adenocarcinoma: a derivation and validation study," Ebiomedicine, vol. 32, pp. 134-141, 2018.

[17] T. Ma, Z.-J. Wu, H. Xu et al., "Nomograms for predicting survival in patients with metastatic gastric adenocarcinoma who undergo palliative gastrectomy," BMC Cancer, vol. 19, no. 1, p. 852, 2019.

[18] L. Zhang, D. Dong, H. Li et al., "Development and validation of a magnetic resonance imaging-based model for the prediction of distant metastasis before initial treatment of nasopharyngeal carcinoma: a retrospective cohort study," Ebiomedicine, vol. 40, pp. 327-335, 2019.

[19] S.-L. Zhang, Z.-M. Wang, W.-R. Wang, X. Wang, and Y.-H. Zhou, "Novel nomograms individually predict the survival of patients with soft tissue sarcomas after surgery," Cancer Management and Research, vol. 11, pp. 3215-3225, 2019.

[20] S. E. Eggener, P. T. Scardino, P. C. Walsh et al., "Predicting 15year prostate cancer specific mortality after radical prostatectomy," Journal of Urology, vol. 185, no. 3, pp. 869-875, 2011.

[21] T. Eguchi, S. Bains, M. C. Lee et al., "Impact of increasing age on cause-specific mortality and morbidity in patients with stage I Non-small-cell lung cancer: a competing risks analysis," Journal of Clinical Oncology, vol. 35, no. 3, pp. 281-290, 2017.

[22] M. J. Wensink, "Size, longevity and cancer: age structure," Proceedings of the Royal Society B: Biological Sciences, vol. 283, no. 1838, Article ID 20161510, 2016.
[23] M. A. Adam, S. Thomas, T. Hyslop, R. P. Scheri, S. A. Roman, and J. A. Sosa, "Exploring the relationship between patient age and cancer-specific survival in papillary thyroid cancer: rethinking current staging systems," Journal of Clinical Oncology, vol. 34, no. 36, pp. 4415-4420, 2016.

[24] A. C. Hesla, P. Tsagozis, N. Jebsen, O. Zaikova, H. Bauer, and O. Brosjö, "Improved prognosis for patients with ewing sarcoma in the sacrum compared with the innominate bones," The Journal of Bone and Joint Surgery, vol. 98, no. 3, pp. 199-210, 2016.

[25] J. T. Park, J.-L. Roh, S.-O. Kim et al., "Prognostic factors and oncological outcomes of 122 head and neck soft tissue sarcoma patients treated at a single institution," Annals of Surgical Oncology, vol. 22, no. 1, pp. 248-255, 2015.

[26] A. Raciborska, K. Bilska, M. Rychlowska-Pruszynska et al., "Internal hemipelvectomy in the management of pelvic Ewing sarcoma-are outcomes better than with radiation therapy?" Journal of Pediatric Surgery, vol. 49, no. 10, pp. 1500-1504, 2014.

[27] Z.-H. Wan, Z.-H. Huang, and L.-B. Chen, "Survival outcome among patients with Ewing's sarcoma of bones and joints: a population-based cohort study," Sao Paulo Medical Journal, vol. 136, no. 2, pp. 116-122, 2018.

[28] S. M. Gayner, J. E. Lewis, and T. V. McCaffrey, "Effect of resection margins on dermatofibrosarcoma protuberans of the head and neck," Archives of Otolaryngology-Head and Neck Surgery, vol. 123, no. 4, pp. 430-433, 1997.

[29] T. I. Yock, M. Krailo, C. J. Fryer et al., "Local control in pelvic ewing sarcoma: analysis from INT-0091-A report from the children's Oncology group," Journal of Clinical Oncology, vol. 24, no. 24, pp. 3838-3843, 2006

[30] D. Donati, J. Yin, C. Di Bella et al., "Local and distant control in non-metastatic pelvic Ewing's sarcoma patients," Journal of Surgical Oncology, vol. 96, no. 1, pp. 19-25, 2007.

[31] M. Okamoto, M. Kito, Y. Yoshimura et al., "The status quo of treatment and clinical outcomes for patients over 80 years of age with high-grade soft tissue sarcoma: report from the soft tissue tumor registry in Japan," Japanese Journal of Clinical Oncology, vol. 48, no. 10, pp. 900-905, 2018.

[32] V. Grünwald, S. Litière, R. Young et al., "Absence of progression, not extent of tumour shrinkage, defines prognosis in soft-tissue sarcoma-an analysis of the EORTC 62012 study of the EORTC STBSG," European Journal of Cancer, vol. 64, pp. 44-51, 2016.

[33] J. D. Stevenson, M. K. Laitinen, M. C. Parry, V. Sumathi, R. J. Grimer, and L. M. Jeys, "The role of surgical margins in chondrosarcoma," European Journal of Surgical Oncology, vol. 44, no. 9, pp. 1412-1418, 2018.

[34] G. K. Zagars, M. T. Ballo, P. W. T. Pisters, R. E. Pollock, S. R. Patel, and R. S. Benjamin, "Surgical margins and reresection in the management of patients with soft tissue sarcoma using conservative surgery and radiation therapy," Cancer, vol. 97, no. 10, pp. 2544-2553, 2003. 